\title{
INTEGRABLE ALMOST TANGENT STRUCTURES
}

\author{
F. BRICKELL \& R. S. CLARK
}

Suppose that $\Gamma$ is a given pseudogroup of local diffeomorphisms $f: R^{n} \rightarrow R^{n}$. A maximal atlas of charts of a manifold $M$ whose changes of coordinates belong to $\Gamma$ determines a $\Gamma$ structure on $M$. These particular charts of $M$ are said to be adapted for the $\Gamma$ structure.

The set $\Gamma_{G}$ of all local diffeomorphisms $f$ whose derivatives $D f$ have values in some given Lie subgroup $G$ of $G L\left(R^{n}\right)$ is an important example of a pseudogroup. A $\Gamma_{G}$ structure on $M$ is called an integrable $G$ structure. Examples of these are integrable almost complex structures and integrable almost tangent structures.

$\Gamma$ structures on manifolds $M$ and $M_{1}$ are said to be isomorphic if there exists a bijection

$$
\phi: M_{1} \rightarrow M
$$

such that $x$ is an adapted chart of $M$ iff $x \circ \phi$ is an adapted chart of $M_{1}$.

Any complex manifold has a standard integrable almost complex structure. A well-known theorem states that any integrable almost complex structure on a manifold $M$ is isomorphic to this standard structure on some complex manifold.

Any tangent manifold has a standard integrable almost tangent structure. But an integrable almost tangent structure on a manifold $M$ is not necessarily isomorphic to this standard structure on some tangent manifold. In this paper we find necessary and sufficient conditions for the existence of such a tangent manifold.

\section{Locally affine structures}

Suppose given a $\Gamma$ structure on a manifold $M$. An atlas of adapted charts of $M$ whose changes of coordinates belong to a given subpseudogroup $\Gamma^{\prime}$ of $\Gamma$ determines a subordinate $\Gamma^{\prime}$ structure on $M$. In general such a subordinate structure does not exist.

A locally affine structure on $M$ is a pseudogroup structure with coordinate transformations of the type

$$
z \rightarrow A z+b,
$$

where $A \in G L\left(R^{n}\right), b \in R^{n}$. A manifold with such a structure carries a standard

Communicated by K. Yano, June 4, 1973. 
flat linear connection whose components with respect to the adapted charts are zero.

A subpseudogroup structure with coordinate transformations of the type

$$
z \rightarrow A z
$$

is a locally centro-affine structure.

A locally affine structure does not necessarily admit a subordinate locally centro-affine structure. In order to be able to state conditions for it to do so we recall [3] that a vector field $V$ on a manifold $M$ is concurrent with respect to a linear connection $\nabla$ on $M$ if

$$
\nabla_{X} V=X
$$

for any vector field $X$ in $M$.

Theorem 1. A locally affine structure on $M$ has a subordinate locally centroaffine structure iff $M$ admits a global vector field concurrent with respect to its flat connetion.

Proof. Suppose that $M$ has a subordinate locally centro-affine structure. For each chart $y$ adapted for this structure we define the local vector field

$$
y^{j} \partial / \partial y^{j}
$$

These local fields agree on the intersection of their domains and so define a global vector field $V$ on $M$. This is concurrent with respect to the flat connection $\nabla$ since, if $X=a^{i} \partial / \partial y^{i}$,

$$
\nabla_{X} V=a^{i} \nabla_{\partial / \partial y^{i}}\left(y^{j} \partial / \partial y^{j}\right)=a^{i} \partial / \partial y^{i}=X .
$$

Conversely, suppose that $M$ carries a concurrent vector field $V$. For each chart $y$ adapted for the locally affine structure let $V=v^{i} \partial / \partial y^{i}$. Then for any vector field $X=a^{i} \partial / \partial y^{i}$

$$
\nabla_{X} V=a^{i} \frac{\partial v^{j}}{\partial y^{i}} \frac{\partial}{\partial y^{j}}
$$

Since this must be $X$ it follows that

$$
v=y+c
$$

for some $c \in R^{n}$. Such functions $v$ are therefore charts adapted for the locally affine structure and so, on any intersection of domains,

$$
\bar{v}=A v+b,
$$

where $A, b$ have values in $G L\left(R^{n}\right)$ and $R^{n}$ respectively. But because $V$ is a 
global vector field, it follows that $b=0$. The charts $v$ therefore define a locally centro-affine structure on $M$ subordinate to the given locally affine structure.

q.e.d.

We shall say that a locally affine structure on a manifold $M$ is complete if the flat connection on $M$ is complete. Any complete locally affine structure on a connected manifold $M$ determines a complete locally affine structure on its simply connected covering manifold $M^{\prime}$. This structure on $M^{\prime}$ is isomorphic to the standard locally affine structure on $R^{n}$ determined by its identity chart [2].

Theorem 2. A locally affine structure on a connected manifold $M$ which

(i) is complete,

(ii) admits a subordinate locally centro-affine structure, is isomorphic to the standard structure on $R^{n}$.

Proof. Theorem 1 shows that $M$ carries a concurrent vector field $V$. This lifts to a concurrent vector field $W$ on $R^{n}$. If $y$ is the identity chart on $R^{n}$, an argument used in the previous proof shows that

$$
W=\left(y^{i}+c^{i}\right) \frac{\partial}{\partial y^{i}}
$$

for some $c^{i} \in R$, and so $W$ has just one zero. This arises from a zero of the vector field $V$. But since $W$ has only one zero, $R^{n}$ must cover $M$ just once.

\section{Integrable almost tangent structures}

A manifold $M$ modelled on $R^{n} \oplus R^{n}$ carries a foliation (of dimension $n$ ) if if it has a pseudogroup structure whose coordinate transformations are local diffeomorphisms of the type

$$
(z, w) \rightarrow(f z, g(z, w))
$$

where $f$ is a local diffeomorphism in $R^{n}$. If $(x, y)$ is an adapted chart at a point $m \in M$, the leaf $F_{m}$ containing $m$ is determined locally by $x=x m$ and it admits $y \mid F_{m}$ as a chart.

An integrable almost tangent structure [1] on $M$ is a pseudogroup structure with coordinate transformations of the type

$$
(z, w) \rightarrow\left(f z,(D f)_{z} w+b z\right)
$$

where $f$ is a local diffeomorphism in $R^{n}$, and the local function $b: R^{n} \rightarrow R^{n}$ is differentiable. An integrable almost tangent manifold carries an underlying foliation $\mathscr{F}$. A chart $(x, y)$ which is adapted for the almost tangent structure is necessarily adapted for $\mathscr{F}$, and the charts $y \mid F_{m}$ determine a locally affine structure on the leaf $F_{m}$.

A subpseudogroup structure with coordinate transformations of the type 


$$
(z, w) \rightarrow\left(f z,(D f)_{z} w\right)
$$

is a nearly tangent structure.

Theorem 3. An integrable almost tangent structure on $M$ has a subordinate nearly tangent structure iff $M$ admits a global vector field which is tangent to the underlying foliation $\mathscr{F}$ and concurrent with respect to the locally affine structure on each leaf of $\mathscr{F}$.

Proof. Suppose that $M$ has a subordinate nearly tangent structure with charts $(x, y)$. The local vector fields $y^{i} \partial / \partial y^{i}$ agree on the intersection of their domains since

$$
y^{i} \frac{\partial}{\partial y^{i}}=y^{i}\left(\frac{\partial \bar{x}^{j}}{\partial y^{i}} \frac{\partial}{\partial \bar{x}^{j}}+\frac{\partial \bar{y}^{j}}{\partial y^{i}} \frac{\partial}{\partial \bar{y}^{j}}\right)=y^{i} \frac{\partial \bar{x}^{j}}{\partial x^{i}} \frac{\partial}{\partial \bar{y}^{j}}=\bar{y}^{i} \frac{\partial}{\partial \bar{y}^{i}},
$$

and together they define a global vector field $\Lambda$ on $M$. This vector field is tangent to the foliation $\mathscr{F}$, and is concurrent with respect to the locally affine structures on the leaves.

Conversely suppose that $M$ carries such a vector field $\Lambda$. For each chart $(x, y)$ adapted for the almost tangent structure let $\Lambda=v^{i} \partial / \partial y^{i}$, where $v^{i}$ depend on $x, y$. Then for any vector field $X=a^{i} \partial / \partial y^{i}$ on a leaf

$$
\nabla_{X} \Lambda=a^{i} \frac{\partial v^{j}}{\partial y^{i}} \frac{\partial}{\partial y^{j}}
$$

Since this must be $X$, it follows that

$$
v=y+c(x)
$$

for some local differentiable function $c: R^{n} \rightarrow R^{n}$. Consequently $(x, v)$ are also charts of $M$ adapted for the almost tangent structure and so on any intersection of their domains

$$
\bar{x}^{i}=f^{i}(x), \quad \bar{v}^{i}=\frac{\partial \bar{x}^{i}}{\partial x^{j}} v^{j}+b^{i}(x)
$$

In terms of such a chart, $\Lambda=v^{i} \partial / \partial v^{i}$, and because $\Lambda$ is a global vector field it follows that $b^{i}=0$. The charts $(x, v)$ therefore define a subordinate nearly tangent structure on $M$. q.e.d.

Suppose that $M$ is a manifold with a nearly tangent structure, and that $\Lambda$ is the associated vector field defined in Theorem 3.

Theorem 4. The set of zeros of $\Lambda$ can be given the structure of a regular submanifold $M^{\prime}$ of $M$ of dimension $n$.

Proof. Let $M^{\prime}$ be the set of zeros of $\Lambda$, and $p$ a given point of $M^{\prime}$. Choose a chart $\xi=(x, y)$ at $p$ adapted for the nearly tangent structure and having range $U \times V \subset R^{n} \oplus R^{n}$, where $U$ and $V$ are open cubes in $R^{n}$. The inter- 
section $M^{\prime} \cap$ (domain $\xi$ ) is just the set of points for which $y=0$.

If $y: M^{\prime} \rightarrow M$ is the natural injection, then $x^{\prime}=x \circ j$ is an injection $M^{\prime} \rightarrow$ $R^{n}$ with range $U$. It is therefore a chart for $M^{\prime}$. If $\bar{x}^{\prime}$ is another chart for $M^{\prime}$ at $p$ obtained from $\bar{\xi}=(\bar{x}, \bar{y})$, then $\bar{x}^{\prime} \circ x^{\prime-1}=\bar{x} \circ x^{-1}$, and this is a local diffeomorphism in $R^{n}$. As $p$ varies over $M^{\prime}$, the charts $x^{\prime}$ obtained in this way define a manifold structure of dimension $n$ on $M^{\prime}$.

The representative for $j$ in terms of the charts $x^{\prime}, \xi$ is the function $z \rightarrow(z, 0)$. Consequently $M^{\prime}$ is a submanifold of $M$. The domain of $x^{\prime}$ is the intersection of the domain of $\xi$ with $M^{\prime}$. This implies that $M^{\prime}$ is a regular submanifold of $M$.

\section{The main theorem}

We shall say that an integrable almost tangent structure on a manifold $M$ is complete if the locally affine structure on each leaf of the foliation $\mathscr{F}$ is complete.

Suppose that such a structure is given on $M$, and that it admits a subordinate nearly tangent structure. Each leaf then admits a subordinate locally centroaffine structure. According to Theorem 2, the locally affine structure on each leaf is isomorphic to the standard structure on $R^{n}$, and therefore admits a global adapted chart $s$.

The vector field $\Lambda$, introduced in Theorem 3 , has just one zero in each leaf. We can therefore define the function $\pi: m \rightarrow p$, where $p$ is the zero in the leaf $F_{m}$. This maps $M$ onto $M^{\prime}$.

Choose a point $p \in M^{\prime}$, and let $j_{p}: F_{p} \rightarrow M$ be the natural injection. Let $\xi=$ $(x, y)$ be a chart of $M$ at $p$ chosen as in the proof of Theorem 4. Then the chart $y \circ j_{p}$ is adapted for the locally centro-affine structure on $F_{p}$ and on its domain

$$
y \circ j_{p}=A s+b
$$

for some $A \in G L\left(R^{n}\right), b \in R^{n}$. The function $A s+b$ is a global chart for the manifold $F_{p}$. It can be shown to be adapted to the locally centro-affine structure on $F_{p}$. This extension of the chart $y_{\circ} j_{p}$ can be carried out for each point $p$ in the open set

$$
W^{\prime}=\left(x^{\prime}\right)^{-1} U
$$

of $M^{\prime}$. Consequently it defines a function $Y$ on $\pi^{-1}\left(W^{\prime}\right)$ with values in $R^{n}$.

The function $x$ is constant on the slices of $\xi$, and so it also can be extended to a function $X$ on $\pi^{-1}\left(W^{\prime}\right)$ with values in $R^{n}$. This is constant on the leaves of $\mathscr{F}$.

Lemma. The function $(X, Y): M \rightarrow R^{n} \oplus R^{n}$ is a chart adapted to the nearly tangent structure on $M$. 
Proof. In the first place we observe that $(X, Y)$ is a local injection onto the open set $U \times R^{n}$.

We shall say that $(X, Y)$ is nearly tangent at a point $m$, if it is defined on some neighborhood of $m$, and if its restriction to this neighborhood is a chart adapted to the nearly tangent structure on $M$. To prove the lemma it is sufficient to show that $(X, Y)$ is nearly tangent at each point of its domain $\pi^{-1}\left(W^{\prime}\right)$.

Let $p$ be a point of $W^{\prime}$, and consider the set $S$ of points in $F_{p}$ at which $(X, Y)$ is nearly tangent. $S$ is not empty because $(X, Y)$ is nearly tangent at all points of the domain of the chart $\xi . S$ is, of course, an open subset of $F_{p}$. We complete the proof of this lemma by showing that $S$ is also a closed subset of $F_{p}$ and will therefore coincide with $F_{p}$.

Choose a point $m$ in the closure of $S$, and then choose a chart $\bar{\xi}=(\bar{x}, \bar{y})$ at $m$, with range $\bar{U} \times \bar{V}$ (where $\bar{U}, \bar{V}$ are open in $R^{n}$ ), adapted for the nearly tangent structure. The domain of $\bar{\xi}$ will meet $S$, and we choose a point $q$ of the intersection. Since $(X, Y)$ is nearly tangent at $q$, there is a neighborhood

$$
W_{1}=\bar{\xi}^{-1}\left(U_{1} \times V_{1}\right)
$$

of $q$, where $U_{1}$ and $V_{1}$ are cubes contained in $\bar{U}$ and $\bar{V}$, such that $(X, Y)$ is defined on $W_{1}$ and its restriction to $W_{1}$ is a chart adapted to the nearly tangent structure. Consequently on $W_{1}$

$$
X^{i}=f^{i}(\bar{x}), \quad Y^{i}=\frac{\partial X^{i}}{\partial \bar{x}^{j}} \bar{y}^{j},
$$

where $f$ is a local diffeomorphism in $R^{n}$ with domain $U_{1}$.

The first relation holds on $\bar{W}=\bar{\xi}^{-1}\left(U_{1} \times \bar{V}\right)$. The second relation also holds on $\bar{W}$ because the functions $Y$ and $\bar{y}$ induce the same locally centro-affine structure on each leaf of $\mathscr{F}$ which meets $W_{1}$. Consequently the function $(X, Y)$ is defined on $\bar{W}$ and its restriction to $\bar{W}$ is a chart adapted to the nearly tangent structure on $M$. But $m \in \bar{W}$ and therefore $m \in S$. It follows that $S$ is a closed subset of $F_{p}$. q.e.d.

Suppose that $M^{\prime}$ is any manifold modelled on $R^{n}$, and that $T M^{\prime}$ is its tangent manifold. Let $\pi^{\prime}: T M^{\prime} \rightarrow M^{\prime}$ be the natural projection. Associated with any chart $x^{\prime}$ of $M^{\prime}$ with domain $W^{\prime}$ we have a standard chart $\left(X^{\prime}, Y^{\prime}\right)$ of $T M^{\prime}$ with domain $\left(\pi^{\prime}\right)^{-1} W^{\prime}$ defined by

$$
X^{\prime}: v \rightarrow x^{\prime} m, \quad Y^{\prime}: v \rightarrow a,
$$

where $v=a^{i}\left(\partial / \partial x^{\prime}\right)_{m}$. These charts define a nearly tangent structure on $T M^{\prime}$ whose underlying integrable almost tangent structure is complete.

Conversely, we have

Theorem 5. An integrable almost tangent structure on a manifold $M$ which (i) is complete, 
(ii) admits a subordinate nearly tangent structure, is isomorphic to the standard structure on a tangent manifold.

Proof. Let $M^{\prime}$ be the submanifold of $M$ defined in Theorem 4. Choose any point $m \in M$ and a chart $\xi=(x, y)$ of $M$ at $p=\pi m$ as in the proof of Theorem 4. The previous lemma shows that this can be extended to a chart $(X, Y)$ at $m$.

Let $x^{\prime}$ be the chart of $M^{\prime}$ at $p$ associated with $\xi$. The local function

$$
m \rightarrow Y^{i}(m)\left(\partial / \partial x^{\prime i}\right)_{p}
$$

is independent of the choice of $\xi$. Such functions determine a bijection $\phi$ between $M$ and $T M^{\prime}$. In terms of the chart $(X, Y)$ of $M$ and the standard chart $\left(X^{\prime}, Y^{\prime}\right)$ of $T M^{\prime}$ associated with $x^{\prime}$, the representative of $\phi$ is the identity function on $U \times R^{n}$. Consequently $\phi$ is a diffeomorphism of $M$ onto $T M^{\prime}$.

Since it maps the atlas of adapted charts $(X, Y)$ of $M$ to the atlas of adapted charts $\left(X^{\prime}, Y^{\prime}\right)$ of $T M^{\prime}, \phi$ is an isomorphism of the integrable almost tangent structures on these manifolds. q.e.d.

A manifold with an integrable almost tangent structure does not necessarily admit any subordinate nearly tangent structure.

To illustrate this, consider the circle $S$ and its atlas $\mathscr{A}$ whose charts are restrictions of the global function $(\cos \alpha, \sin \alpha) \rightarrow \alpha$. Two such charts differ by a coordinate transformation given locally by

$$
z \rightarrow z+c
$$

for some $c \in R$, and so $\mathscr{A}$ determines a locally affine structure $\Gamma_{\mathscr{A}}$ on $S$. This structure is complete. Now consider the torus $T=S \times S$. The atlas of charts $x \times y$, where $x, y \in \mathscr{A}$, defines an integrable almost tangent structure $\Sigma$ on $T$. The leaves of the foliation $\mathscr{F}$ are the circles $F_{p}=p \times S$. The locally affine structure on any leaf is isomorphic to $\Gamma_{\mathscr{A}}$ and so it is complete. Since $T$ is compact, it cannot be a tangent manifold. It follows from Theorem 5 that the integrable almost tangent structure $\Sigma$ cannot admit any subordinate nearly tangent structure.

\section{References}

[ 1 ] R. S. Clark \& M. R. Bruckheimer, Sur les structures presque tangents, C. R. Acad. Sci. Paris 251 (1960) 627-629.

[2] J. A. Wolf, Spaces of constant curvature, McGraw-Hill, New York, 1967, Chapter I, §1.9.

[3] K. Yano, The theory of Lie derivatives and its applications, North-Holland, Amsterdam, 1955, 168. 
